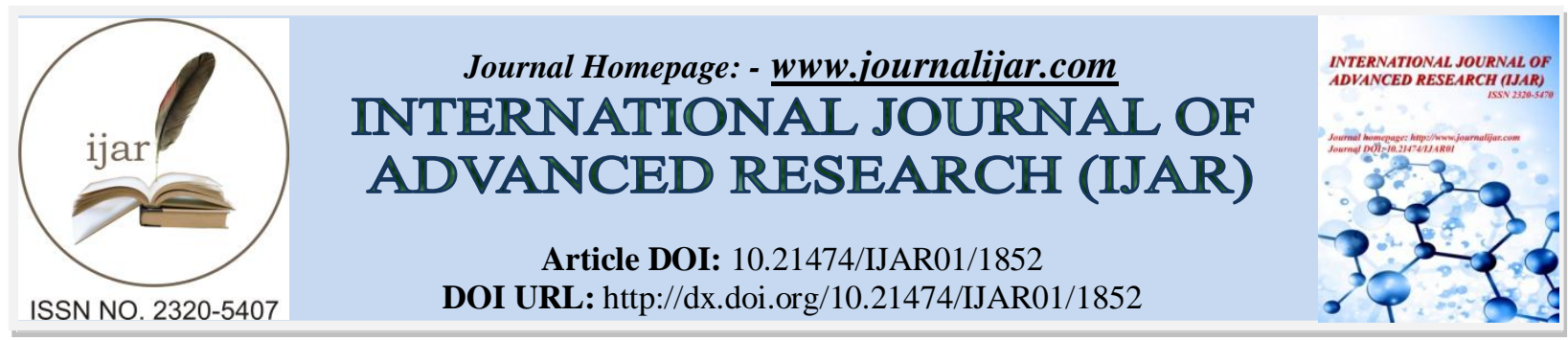

RESEARCH ARTICLE

\title{
A COMPARISON OF VARIOUS VARIANCE REDUCTION TECHNIQUES VIS A VIS IMPORTANCE SAMPLING TO IMPROVE MONTECARLO SIMULATION.
}

Sunny Babber and Parmil Kumar.

Department of Statistics, University of Jammu, Jammu-India-180006.

\section{Manuscript Info}

Manuscript History

Received: 12 August 2016

Final Accepted: 22 September 2016

Published: October 2016

Key words:-

Montecarlo Simulation; Variance

Reduction; Antithetic Variable; Control

Variable; Importance Sampling.

\section{Abstract}

With the rapid increase in computer technology and power, we need to develop such a technique which perform fast simulation and provide better result in least possible time. The Monte-Carlo (MC) simulation is widely used technique for simulation to obtain the results. By using MC technique, one get approximate answer quickly to a higher level of accuracy. But the major worrisome thing about the MC simulation is that, it provides approximation to the desired answer than the exact requirement which results an approximate error. Approximate error is the major factor taken into account whenever we evaluate answers. These approximation errors always effect the estimation of performance measure in Monte-Carlo simulation to a greater extent. In this paper, we discussed various variance reduction technique to overcome such type of error and show that how the Importance Sampling (IS) technique provide better approximation by reducing the variance to great extent as compared to other well-known techniques viz., Antithetic variable and Control Variate.

Copy Right, IJAR, 2016,. All rights reserved.

\section{Introduction:-}

The word "Simulation" means imitation of the operation of real world process or system over a time [4]. In early days, simulation was just used to understand deterministic problems and statistical sampling was used to estimate the uncertainties associated with simulation but when Monte Carlo simulation came into an existence it reinvented the old simulation approach to solve deterministic problems using probabilities analogy [14].

G.S. Fishman (1996) defined the Monte-Carlo Simulation as a method based on probability analysis and is done by running a number of different variables through a model in order to determine the different outcomes. With the increase in computer technology and power, we need to develop such a technique which perform fast simulation and provide better result in short span of time. By using this method, we get approximate answer quickly and to a higher level of accuracy $[8,10]$. The major thing is that MC simulation only provides approximation to our answer than exact answer itself. This leads to approximate error. This error is a major factor to evaluate answers obtained using these methods $[2,7]$. These approximation errors always effect the estimation of performance measure in MonteCarlo simulation to greater extent. Thus introduce Variance Reduction Techniques (VRT) [13] which provides the better estimated result by utilizing known information about the simulation model, the more we know about the behaviour of system, the greater amount of variance reduction can be achieved. Even a simple variance reduction method often is remarkable, effective and easy to implement.

Corresponding Author:- Sunny Babber.

Address:- Department of Statistics, University of Jammu, Jammu-India-180006. 
In this paper, we discussed some of the Variance Reduction Techniques to overcome approximate error and show that how the Importance Sampling (IS) Technique provide better approximation by reducing the variance to great extend as compared to other well-known techniques viz., Antithetic variable and Control Variate. The rest of the paper is organized as follows. In Section 1.2 we introduce some Variance Reduction Techniques. In section 1.3 we take simple network diagram as example. In section 1.4-1.6, we discuss Antithetic Variable, Control Variate and Importance Sampling respectively, and obtained results by using MATLAB Program. In section 1.7, we compare the obtained numerical results and give the conclusion.

\section{Variance reduction:-}

Variance Reductions Techniques (VRT) are basically used to improve the efficiency of MC simulation by utilizing the known information about the simulation model. The more we know about the behavior of the system the greater amount of reduction in variance is achieved.

The main variance reduction techniques that we are discussed in this paper are given below

1. Antithetic Random Variable.

2. Control Variate.

3. Importance Sampling (IS).

In this paper, we have considered a Simple Network Diagram to study and compare all the above variance reduction techniques. A MATLAB program has been coded for comparison of results. We begin with estimation of the results using Crude Monte Carlo (CMC) Method.

Consider a simple undirected Network graph as shown in fig.1

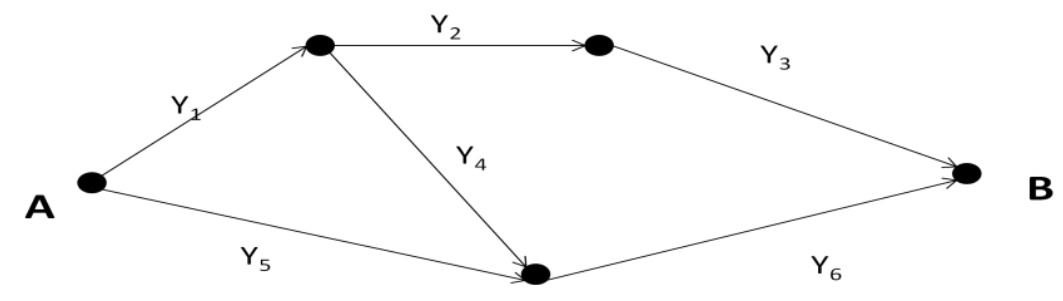

Figure 1:- Different length of the path from A to B.

Let the random variables $Y_{1}, Y_{2}, Y_{3}, Y_{4}, Y_{5}, Y_{6}$ are the length of each edge of Network Diagram and suppose we have to find the expected length $\mathrm{L}$ of the shortest path from Node A to Node $\mathrm{B}$,

That is, $\quad L=E[G(Y)]$

Where

$$
G(Y)=\min \left[Y_{1}+Y_{2}+Y_{3}, Y_{1}+Y_{4}+Y_{6}, Y_{5}+Y_{6}\right]
$$

Note that, $G(Y)$ is a non-decreasing function in each component vector $Y$. here we suppose that each of the length of $Y_{i}, i=1,2,3,4,5,6$ is independent and follows uniform distribution with parameter 0 and $d_{i}$, i.e. $Y_{i} \sim U\left(0, d_{i}\right), i=$ $1,2,3,4,5,6$ with arbitrary $\left(d_{1}, d_{2}, d_{3}, d_{4}, d_{5}, d_{6}\right)=(1,2,1,4,2,3)$

Let $Y_{i}=d_{i} V_{i}, i=1,2,3,4,5,6$, here we restate the original problem into the problem of estimation and is given by

$$
L=E[g(V)]
$$

where, $V=\left(V_{1}, V_{2}, V_{3}, V_{4}, V_{5}, V_{6}\right)$ and $\mathrm{g}(\mathrm{V})=G\left(d_{1} V_{1}, d_{2} V_{2}, d_{3} V_{3}, d_{4} V_{4}, d_{5} V_{5}, d_{6} V_{6}\right)$

Crude Monte Carlo (CMC) generate a random vector $V_{1}, V_{2}, V_{3}, V_{4}, V_{5}, V_{6}$, each are independent and follows uniform distribution with parameter $(0,1)$ i.e. $V_{i} \sim U(0,1)^{6}$ and returning a value

$$
\widehat{L}=\frac{1}{N} \sum_{k=1}^{N} g\left(V_{K}\right)
$$


as an estimate of $L$. To test the accuracy of this estimate $\widehat{L}$, we need to look at the probability distribution of the estimator $\hat{L}$. In particular $\hat{L}$, is an unbiased estimator of $L$ and $\operatorname{Var}(\hat{L})$ is $\frac{\sigma^{2}}{N}$, where $\sigma^{2}$ is the variance of $g\left(V_{K}\right)$. Hence if $\sigma^{2}$ is finite, then $\operatorname{Var}(\hat{L}) \rightarrow 0$ as $N \rightarrow \infty$.

By using the CLT, we have $\hat{L} \sim N\left(L, \frac{\sigma^{2}}{N}\right)$.

This implies that, for large $N$,

$$
P\left\{\frac{|\tilde{L}-L|}{\sigma / \sqrt{N}}>Z_{1-\alpha / 2}\right\} \approx \alpha
$$

where, $Z_{1-\alpha / 2}$ is the $(1-\alpha / 2)$ quantile of the standard normal distribution.

Since, Sample Variance given by

$$
\mathrm{S}^{2}=\frac{1}{\mathrm{~N}-1} \sum_{\mathrm{i}=1}^{\mathrm{N}}\left[\mathrm{g}\left(\mathrm{V}_{\mathrm{i}}\right)-\hat{\mathrm{L}}\right]^{2}
$$

is an unbiased estimator of $\sigma^{2}$. So we replace $\sigma^{2}$ by $S^{2}$.

Thus the equation (1.5) is rewritten as

$$
P\left\{\hat{L}-Z_{1-\alpha / 2} S / \sqrt{N} \leq L \leq \hat{L}+Z_{1-\alpha / 2} S / \sqrt{N}\right\} \approx \alpha
$$

Here $\left(\hat{L}-Z_{1-\alpha / 2} S / \sqrt{N}, \hat{L}+Z_{1-\alpha} / 2 S / \sqrt{N}\right)$ is nothing but approximate $\alpha$-confidence interval for estimating $L$. Typical values of $\alpha$ are 0.90 and 0.95 with corresponding quartiles 1.65 and 1.96 . The accuracy of an estimate $\hat{L}$ is often reported by its estimated relative error $\frac{\mathrm{S}}{(\sqrt{\mathrm{N}} / \widehat{\mathrm{L}})}$. Using MATLAB program for CMC simulation, we attained a typical estimated value of $\hat{L}=1.6827$ with an estimated Relative Error (RE) is 0.4068 and Elapsed time is 0.047000 seconds.

\section{Antithetic Variables:-}

A real valued pair is said to be Antithetic pair, if they have identical probability distribution and are negatively correlated [8]. If original function gives high value it provides low and vice-versa. As we know that MC averages are used for reduction in error which occur in algorithm due to randomness. By making use of Antithetic variable we reduce further some more part of error which was ignored by CMC. In other words, An Antithetic sample is that which is opposite to the original function. Graphically, some samples and their antithetic counterparts are shown below in fig. 2.
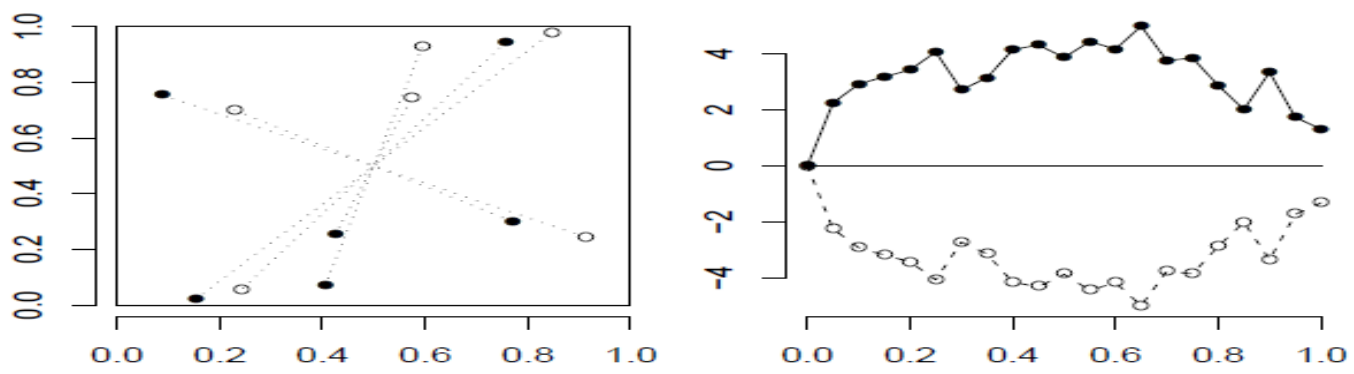

Figure 2:- The left panel shows 6 points as solid points connected to their antithetic counterpart shown as open circles and the right panel shows one random trajectory of 20 points joined by solid lines connected to the origin along with its antithetic mirror image in open points.

Mathematically speaking, If $X_{k} \sim U(0,1)$, thus their Antithetic counterpart is $\tilde{X}_{k}=1-X_{k}$ and if $X_{k} \sim N(0, \Sigma)$ then their antithetic counterpart is $\tilde{X}_{k}=-X_{k}$ because in this case we use inverse transformation, to get this result. In this paper, we use uniform distribution to solve our problems i.e. when $X_{k} \sim U(0,1)$ then $\tilde{X}_{k}=1-X_{k}$ is antithetic variable. 
Thus the antithetic estimator is given by $\hat{L}_{A}=\frac{1}{N} \sum_{k=1}^{N / 2}\left[f\left(X_{k}\right)+f\left(\tilde{X}_{k}\right)\right]$.

where $\hat{L}_{A}$ is also an unbiased estimator of $\hat{L}=E(Y)$.

Now, the variance of antithetic estimator is given by

$$
\begin{aligned}
\operatorname{var}\left(\hat{L}_{A}\right) & =\operatorname{var}\left\{\frac{1}{N} \sum_{k=1}^{N / 2}\left[f\left(X_{k}\right)+f\left(\tilde{X}_{k}\right)\right]\right\} \\
& =\frac{N / 2}{N^{2}}\left\{\operatorname{var}\left[f\left(X_{k}\right)\right]+\operatorname{var}\left[f\left(\tilde{X}_{k}\right)\right]+2 \operatorname{cov}\left[f\left(X_{k}\right), f\left(\tilde{X}_{k}\right)\right]\right\}
\end{aligned}
$$

But $\operatorname{var}\left[f\left(X_{k}\right)\right]=\operatorname{var}\left[f\left(\tilde{X}_{k}\right)\right]$

Therefore equation (1.10) becomes

$$
\begin{array}{r}
\operatorname{var}\left(\hat{L}_{A}\right)=\frac{1}{2 N}\left\{2 \operatorname{var}\left[f\left(X_{k}\right)\right]+2 \operatorname{cov}\left[f\left(X_{k}\right), f\left(\tilde{X}_{k}\right)\right]\right\} \\
=\frac{\operatorname{var}\left[f\left(X_{k}\right)\right]}{N}\left\{1+\rho\left[f\left(X_{k}\right), f\left(\tilde{X}_{k}\right)\right]\right\}
\end{array}
$$

where

$\rho\left[f\left(X_{k}\right), f\left(\tilde{X}_{k}\right)\right]=\operatorname{corr}\left[f\left(X_{k}\right), f\left(\tilde{X}_{k}\right)\right]$ is the correlation coefficient between antithetic pair $\left[f\left(X_{k}\right), f\left(\tilde{X}_{k}\right)\right]$.

On comparing equation (1.11) with the variance obtained by the CMC estimator i.e. $\frac{\sigma^{2}}{N}$, we see that variance of antithetic variable is smaller by a quantity $\left\{1+\rho\left[f\left(X_{k}\right), f\left(\tilde{X}_{k}\right)\right]\right\}$ and the amount of reduction in variance is depend crucially on negative correlation between the antithetic variable.

Generally, the simulation run provide an output of the form $X_{k}=h(V)$, where $\mathrm{h}$ is a real valued function and $V=\left(V_{1}, V_{2}, V_{3}, V_{4}, V_{5}, V_{6}\right)$ is a random vector of form $U(0,1)$. Suppose we choose another vector $\tilde{X}_{k}$ from i.i.d. $U(0$, 1 ), which is dependent upon $X_{k}$, in such a way that $X_{k}$ and $\tilde{X}_{k}$ are negatively correlated. Then the pair $\left(X_{k}, \tilde{X}_{k}\right)$ is said to be antithetic pair.

Algorithm 1: Step by step Algorithm for estimating Antithetic variable for monotone function is given below:-

Here, we use $X_{k}$ for $f\left(X_{k}\right)$ and $\tilde{X}_{k}$ for $f\left(\tilde{X}_{k}\right)$ (for simplicity)

1. Generate $X_{1}=h\left(V_{1}\right), X_{2}=h\left(V_{2}\right) \ldots \ldots \ldots \ldots X_{N} / 2=h\left(V_{N}\right)$

Then $\tilde{X}_{1}=h\left(1-V_{1}\right), \tilde{X}_{2}=h\left(1-V_{2}\right) \ldots \ldots \ldots \ldots \tilde{X}_{N / 2}=h\left(1-V_{N / 2}\right)$

2. Compare the sample covariance matrix for pair $\left(X_{k}, \tilde{X}_{k}\right)$

$$
C=\left[\begin{array}{cc}
\frac{1}{\left({ }^{N} / 2^{-1}\right)} \sum_{k=1}^{N / 2}\left(X_{k}-\bar{X}\right)^{2} & \frac{1}{N / 2} \sum_{k=1}^{N / 2}\left(X_{k}-\bar{X}\right)\left(\tilde{X}_{k}-\overline{\tilde{X}}\right) \\
\frac{1}{N / 2} \sum_{k=1}^{N / 2}\left(X_{k}-\bar{X}\right)\left(\tilde{X}_{k}-\overline{\tilde{X}}\right) & \frac{1}{\left({ }^{N} / 2^{-1}\right)} \sum_{k=1}^{N / 2}\left(\tilde{X}_{k}-\overline{\tilde{X}}\right)^{2}
\end{array}\right]
$$

3. The estimated value of antithetic variable is given by $\hat{L}_{A}=\frac{1}{N} \sum_{k=1}^{N / 2}\left(X_{k}+\tilde{X}_{k}\right)$

4. The $(1-\alpha)$ confidence interval of $\hat{L}_{A}$ is given by $\left(\hat{L}_{A} \pm Z_{1-\alpha / 2} S . E\right)$

Where, S.E is the estimated standard error and is given by $S . E=\sqrt{\frac{C_{11}+C_{22}+2 C_{12}}{2 N}}$, where $\mathrm{C}_{\mathrm{ij}}$ 's are the element of matrix $\mathrm{C}$ and $Z_{\alpha}$ denotes the $\alpha$-quantile of $N(0,1)$ distribution.

Using $N=10^{4}$, we get the estimated antithetic variable $\hat{L}_{A}=1.6815$, with estimated relative error 0.2834 , Correlation $=-0.5224$ and $95 \%$ Confidence Interval: $(-89.556,92.919)$ and Elapsed time is 0.019000 seconds. 


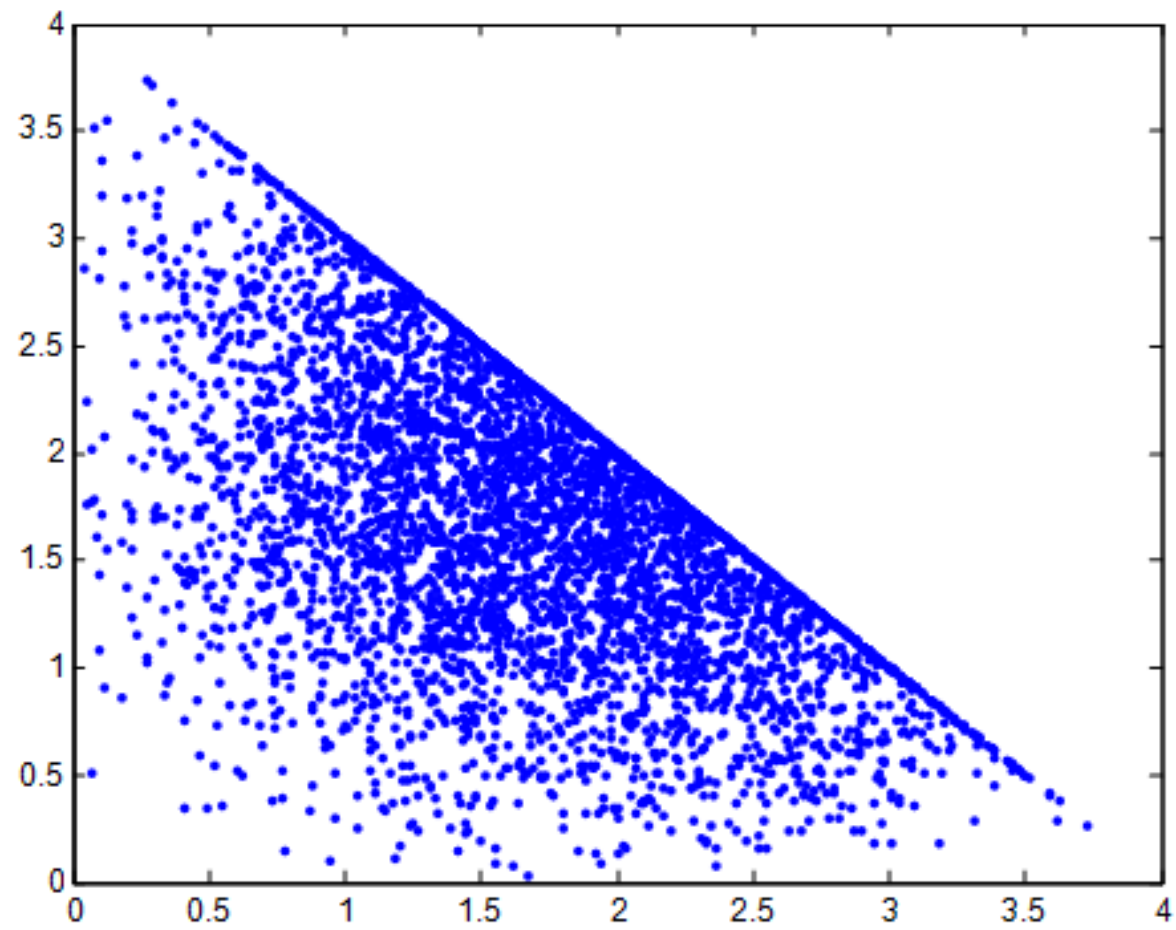

Figure 3:- This figure shows the high negative correlation between $h(V)$ and $h(1-V)$, which means there is big reduction in simulation effort when compared to CMC.

\section{Control Variate:-}

The Control Variable Method [9] is another important and most common variance reduction technique used to reduce the variance of an estimate. Let $X_{k}$ be an unbiased estimator obtain from simulation run. A random variable $X_{k}^{\prime}$, obtained from same simulation run, is said to be control variable of $X_{k}$ if the variable $X_{k}$ and $X_{k}^{\prime}$ are correlated and the expectation of $X_{k}^{\prime}$ is known in advance.

Let $X_{1}, X_{2}, X_{3}, \ldots \ldots \ldots X_{N}$ be the output of $N$ independent simulation runs and let $X_{1}^{\prime}, X_{2}^{\prime}, \ldots \ldots \ldots X_{N}^{\prime}$ are the corresponding control variable with $E\left(X_{k}^{\prime}\right)=\theta$ known.

Here we consider a linear control estimator

$$
\hat{L}_{C}=X_{k}-\lambda\left[X_{k}^{\prime}-E\left(X_{k}^{\prime}\right)\right] \text {, Where, } \lambda \text { is a scalar quantity. }
$$

The above equation can be rewritten as

$$
\hat{L}_{C}=X_{k}-\lambda\left(X_{k}^{\prime}-\theta\right)
$$

The variance of $\hat{\mathrm{L}}_{\mathrm{C}}$ is given by

$$
\begin{aligned}
\operatorname{var}\left(\hat{L}_{C}\right) & =\operatorname{var}\left\{X_{k}-\lambda\left[X_{k}^{\prime}-\theta\right]\right\} \\
& =\operatorname{var}\left(X_{k}\right)+\lambda^{2} \operatorname{var}\left(X_{k}^{\prime}-\theta\right)-2 \lambda \operatorname{cov}\left(X_{k}, X_{k}^{\prime}-\theta\right)
\end{aligned}
$$

Since $\Theta$ is an unbiased estimator of $X_{k}^{\prime}$

$$
\text { Therefore, } \quad \operatorname{var}\left(\hat{L}_{C}\right)=\operatorname{var}\left(X_{k}\right)+\lambda^{2} \operatorname{var}\left(X_{k}^{\prime}\right)-2 \lambda \operatorname{cov}\left(X_{k}, X_{k}^{\prime}\right)
$$

To find the minimum value of $\lambda$, we differentiate equation (1.15) with respect to $\lambda$ and after equating its derivative to zero we obtained

$$
\begin{aligned}
& \frac{d \operatorname{var}\left(\hat{L}_{C}\right)}{d \lambda}=0 \\
& \lambda=\frac{\operatorname{cov}\left(X_{k}, X_{k}^{\prime}\right)}{\operatorname{var}\left(X_{k}^{\prime}\right)}
\end{aligned}
$$

After substituting the value of $\lambda$ in equation (1.15), we get

$$
\begin{aligned}
\operatorname{var}\left(\hat{L}_{C}\right) & =\operatorname{var}\left(X_{k}\right)+\left[\frac{\operatorname{cov}\left(X_{k}, X_{k}^{\prime}\right)}{\operatorname{var}\left(X_{k}^{\prime}\right)}\right]^{2} \operatorname{var}\left(X_{k}^{\prime}\right)-2 \frac{\operatorname{cov}^{2}\left(X_{k}, X_{k}^{\prime}\right)}{\operatorname{var}\left(X_{k}^{\prime}\right)} \\
& =\operatorname{var}\left(X_{k}\right)+\frac{\operatorname{cov}^{2}\left(X_{k}, X_{k}^{\prime}\right)}{\operatorname{var}\left(X_{k}^{\prime}\right)}-2 \frac{\operatorname{cov}^{2}\left(X_{k}, X_{k}^{\prime}\right)}{\operatorname{var}\left(X_{k}^{\prime}\right)}
\end{aligned}
$$




$$
\begin{array}{r}
=\operatorname{var}\left(X_{k}\right)\left\{1-\left[\frac{\operatorname{cov}\left(X_{k}, X_{k}^{\prime}\right)}{\sqrt{\operatorname{var}\left(X_{k}\right)} \sqrt{\operatorname{var}\left(X_{k}^{\prime}\right)}}\right]^{2}\right\} \\
\operatorname{var}\left(\hat{L}_{C}\right)=\operatorname{var}\left(X_{k}\right)\left[1-\rho\left(X_{k}, X_{k}^{\prime}\right)\right]
\end{array}
$$

Where $\rho\left(X_{k}, X_{k}^{\prime}\right)$ is the correlation coefficient between $X_{k}$ and $X_{k}^{\prime}$

Here, from equation (1.18), we can easily see that, if the value of $\rho$ is larger then there will be greater reduction in variance.

\section{Algorithm 2: Step by step Algorithm for estimating control variate:-}

1. Generate $X_{1}, X_{2}, \ldots \ldots \ldots, X_{N}$ from $\mathrm{n}$ independent simulation runs and control variable $X_{1}^{\prime}, X_{2}^{\prime}, \ldots \ldots \ldots, X_{N}^{\prime}$

2. Compute the correlation between $X_{k}$ and $X_{k}^{\prime}$

$$
\mathrm{C}=\left[\begin{array}{cc}
\frac{1}{(N-1)} \sum_{k=1}^{N}\left(X_{k}-\bar{X}_{k}\right)^{2} & \frac{1}{(N-1)} \sum_{k=1}^{N}\left(X_{k}-\bar{X}_{k}\right)\left(X_{k}^{\prime}-\bar{X}_{k}^{\prime}\right) \\
\frac{1}{(N-1)} \sum_{k=1}^{N}\left(X_{k}-\bar{X}_{k}\right)\left(X_{k}^{\prime}-\bar{X}_{k}^{\prime}\right) & \frac{1}{(N-1)} \sum_{k=1}^{N}\left(X_{k}^{\prime}-\bar{X}_{k}^{\prime}\right)^{2}
\end{array}\right]
$$

where, $\quad C=\left[\begin{array}{ll}C_{11} & C_{12} \\ C_{21} & C_{22}\end{array}\right]$

$$
C_{11}=\frac{1}{(N-1)} \sum_{k=1}^{N}\left(X_{k}-\bar{X}_{k}\right)^{2}, C_{12}=C_{21}=\frac{1}{(N-1)} \sum_{k=1}^{N}\left(X_{k}-\bar{X}_{k}\right)\left(X_{k}^{\prime}-\bar{X}_{k}^{\prime}\right) \text { and } C_{22}=\frac{1}{(N-1)} \sum_{k=1}^{N}\left(X_{k}^{\prime}-\bar{X}_{k}^{\prime}\right)^{2}
$$

3. Estimate $\theta$ via control variable estimator $\hat{L}_{C}$

Here $\lambda=\frac{\operatorname{cov}\left(X_{k}, X_{k}^{\prime}\right)}{\operatorname{var}\left(X_{k}^{\prime}\right)}$, which implies $\lambda=\frac{C_{12}}{C_{22}}$ in terms of $\mathrm{C}$ matrix.

4. Determine the (1- $\alpha)$ confidence interval $\left(\widehat{L}_{C} \pm Z_{1-\alpha / 2} S . E\right)$

where, $\quad \operatorname{Standard} \operatorname{Error}(S . E)=\sqrt{\frac{1}{N}\left(1-\frac{C_{12}{ }^{2}}{C_{11} C_{22}}\right)}$

\section{Control Variate Estimation of Stochastic Network Diagram:-}

Here we consider the same problem as used earlier in this chapter to explain the concept of control variable estimation i.e. fig 1 . Using our current parameter $d=(1,2,1,4,2,3)$, we can easily seen that our control variable is given by

$$
X_{k}^{\prime}=\min \left\{Y_{1}+Y_{2}+Y_{3}, Y_{5}+Y_{6}\right\}
$$

i.e. the shortest path will have a length equal to $\mathrm{X}_{\mathrm{k}}^{\prime}$ or most likely it will be $Y_{1}+Y_{2}+Y_{3}$, so that the latter would also be useful as a control variable. A little calculation gives us the expected value of $X_{k}^{\prime}=1.7011$.

Here for a typical value $\mathrm{N}=10^{4}$, estimated value of control variable is $\hat{L}_{C}=1.68136, \mathrm{RE}$ is 0.2620 and $95 \%$ Confidence Interval: $(1.82272,1.82512)$, Elapsed time is 0.047000 seconds

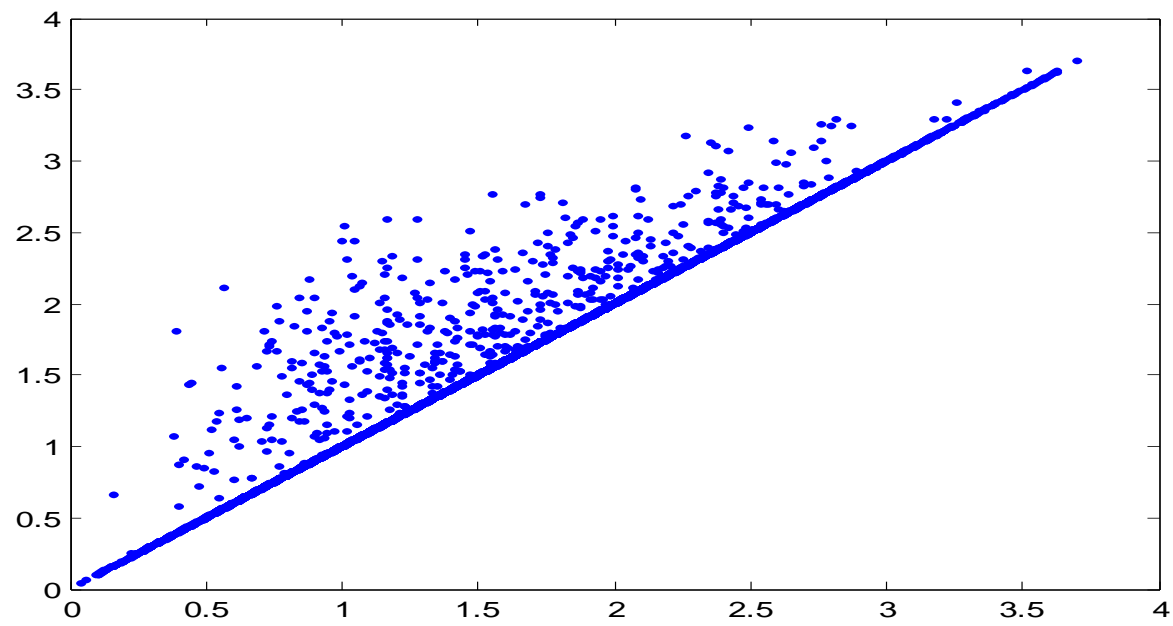

Figure 4:- Shows that there is highly positive correlation coefficient between $X_{k}$ and $X_{k}^{\prime}$ 


\section{Importance Sampling:-}

Importance sampling [2] is the fundamental variance reduction technique used to improve MC Simulation. To explain IS technique let us consider the standard estimation quantity, as before in this paper,

$$
L=E_{f}[G(Y)]=\int G(y) f(y) d y
$$

Here $G(Y)$ is a real valued performance function and $f$ is probability density function of random variable $Y$. Here in equation (1.19) we use the subscript $f$ to indicate that the above expectation is with respect to density $f$.

Let us consider another probability density function $h$ such that $G f$ is dominated by $h$, i.e. $h(y)=0 \Rightarrow G(y) f(y)=$ 0 .

Therefore equation (1.19) is rewritten as

$$
\begin{aligned}
& L=\int G(y) \frac{f(y)}{h(y)} h(y) d y \\
& L=E_{h} G(y) h(y)
\end{aligned}
$$

In equation (1.21) subscript $h$ means that, the expectation is w.r.t $h$, which is called IS density or Proposal density or Instrumental density (as $h$ is used as an instrument to obtain information about $L$ ).

If $Y_{1}, Y_{2}, \ldots \ldots \ldots, Y_{N}$ are i.i.d. random variable with density function $h$ then the unbiased estimator of $L$ given by $\widehat{L}_{I S}=\frac{1}{N} \sum G(Y) \frac{f(y)}{h(y)}$ is called IS estimator, which can also be written as

$$
\hat{L}_{I S}=\frac{1}{N} \sum G(Y) l(y)
$$

Where $l(y)=\frac{f(y)}{h(y)}$, is called likelihood ratio of $f$ and $h$. In particular if $f(y)=h(y)$ (i.e. no change of measure) then IS estimator become CMC estimator and provide the same result as in case of CMC. The choice of IS density $h$ is so crucial for the variance of the estimator $\widehat{L}_{I S}$ in (1.22), next we consider the problem of minimizing the variance of an estimator $\hat{L}_{I S}$ with respect to $h$, that is

The solution of (1.23) is given by

$$
\min _{h} \operatorname{var}_{h}\left\{G(Y) \frac{f(y)}{h(y)}\right\}
$$

if $|G(y)| \geq 0$, then

$$
h^{*}(y)=\frac{|G(y)| f(y)}{\int|G(y)| f(y)} d y
$$

$$
h^{*}(y)=\frac{G(Y) f(y)}{L}
$$

and

$$
\operatorname{var}_{h^{*}}\left(\widehat{L}_{I S}\right)=\operatorname{var}_{h^{*}}(G(Y) l(y))=\operatorname{var}_{h^{*}}(L)=0 .
$$

Therefore the density $g^{*}$ in (1.24) and (1.25) is called optimal importance sampling density.

Algorithm 3: Step by step Algorithm for estimation IS is given by:-

1. First of all select an importance sampling density $h$ that dominates $G f$.

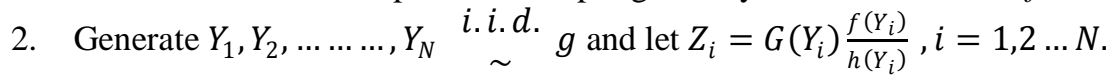

3. Estimate $\hat{L}_{I S}=\frac{1}{N} \sum G(Y) \frac{f(y)}{h(y)}$ via $L$.

4. Determine the $(1-\alpha)$ confidence interval as $\left(\hat{L}_{I S} \pm Z_{1-\alpha / 2} S / \sqrt{N}\right)$.

where $Z_{\alpha}$ denotes the $\alpha$-quantile of $N(0,1)$ distribution and $S$ is the sample standard deviation of $Z_{1}, Z_{2}, \ldots \ldots \ldots, Z_{N}$.

Importance Sampling Estimation of Stochastic Network Diagram:-

We know that the expected value shortest path of undirected network diagram as shown in figure 1, is given by

$$
L=E[G(V)]=\int G(v) d v
$$

Where $V=\left(V_{1}, V_{2}, V_{3}, V_{4}, V_{5}, V_{6}\right)$ and each $V_{1}, V_{2}, V_{3}, V_{4}, V_{5}, V_{6} \underset{\sim}{\text { i.i.d }} U(0,1)$.

Thus its p.d.f is given by

$$
f(v)=1, v \in(0,1)^{6}
$$


Here we suppose that the p.d.f of IS, that is under $\mathrm{g}$ is given by

$$
\begin{aligned}
& h(v)=\prod_{i=1}^{6} u_{i} v_{i}^{u_{i}-1} \\
& \text { where each } V_{i} \stackrel{\text { ind. }}{\sim} \operatorname{Beta}\left(u_{i}, 1\right) \text {, for some } u_{i}>0, i=1,2, \ldots 6 \text {. }
\end{aligned}
$$

Here we can easily generate vector $\mathrm{V}$ under IS by using inverse transformation and a good choice of $u_{i}$ will always provide better result. MATLAB program is used for estimation of $\mathrm{L}$ using IS, for $\mathrm{N}=10^{4}$ and using $\left(v_{1}, v_{2}, v_{3}, v_{4}, v_{5}, v_{6}\right)=(1.1,1.3,1.1,1,1.1,1.3)$ we get a typical estimated value i.e. $\hat{L}_{I S}=1.6776$ with an estimated relative error is 0.2363 and elapsed time is 0.047000 seconds.

\section{Conclusion:-}

In this paper we show that how we can improve the MC Simulation by using some important Variance Reduction Techniques. From the following table we can easily see that the CMC method gives us an estimated value 1.6827 with estimated relative error of 0.4068. Importance Sampling (IS) technique provide better approximation by reducing the variance to great extend as compared to other variance reduction techniques i.e. Antithetic variable and Control Variate. When dealing with rare event probabilities AV and CV techniques not much of practical utility but IS technique leads to the dramatic reduction in variance sometimes up to millions as compared to just 10 times reduction by $\mathrm{AV}$ and $\mathrm{CV}$.

\begin{tabular}{|l|c|c|c|}
\hline \multicolumn{1}{|c|}{$\begin{array}{c}\text { Variance Reduction } \\
\text { Techniques }\end{array}$} & Estimates & Relative Error (\%) & Time Elapsed (sec) \\
\hline Crude Montecarlo & 1.6827 & 0.4068 & 0.334700 \\
\hline Antithetic Variable & 1.6815 & 0.2834 & 0.119000 \\
\hline Control Variable & 1.6813 & 0.2620 & 0.047000 \\
\hline Importance Sampling & 1.6776 & 0.2363 & 0.047000 \\
\hline
\end{tabular}

\section{References:-}

1. Anderson, D.F. and Higham, D.J. (2012): Multilevel Monte Carlo for continuous time Markov chains, with applications in biochemical kinetics. Multiscale Modeling \& Simulation, 10(1):146-179.

2. Asmussen, D.P. Kroese, and R.Y. Rubinstein (2005): Heavy tails, importance sampling and cross-entropy. Stochastic Models, 21 (1):57-76.

3. Avramidis A. N. And Wilson J. R. (1993): A splitting scheme for control variate. Operational Research Letters, 14:187-198.

4. Boyle P., Broadie M., and Glasserman P. (1997): Monte Carlo methods for security pricing. Journal of economic dynamics and control, 21(8):1267-1321.

5. Burgos S. And Giles M. B. (2012): Computing Greeks using multilevel path simulation. In Monte Carlo and Quasi-Monte Carlo Methods 2010, pages 281-296. Springer.

6. Chan J.C.C, Glynn P. W., Kroese D. P., (2011). A comparison of cross-entropy and variance minimization strategies. Journal of Applied Probability. 48A, 183-194.

7. G.S Fishman, (1996): Monte Carlo- concepts, algorithms, and applications. Springer-Verlag New York, Inc.

8. Hammersley J. M. and Morton K. W. (1956): A new Monte Carlo technique: antithetic variable. Mathematical proceedings of the Cambridge philosophical society, 52(3):449-475.

9. Hesterberg T. C. And Nelson B. L. (1998). Control Variate for probability and quantile estimation. Management Science, 44(9):1295-1312.

10. Kahn H. And Marshall A. (1953): Methods of reducing sample size in Monte Carlo computations. Journal of the Operational Research Society of America, 1(5):263-278.

11. Karr A. F. (1993): Probability. Springer, New York.

12. Lumney P. D. and Anderson C. A. (2009): Investigation of the statistical power of the content uniformity tests using simulation studies. Journal of pharmaceutical Innovation, 4:24-35.

13. Wilson J. R. (1984): Variance reduction techniques for digital simulation. American Journal of Mathematical and Management sciences, 4(3):277-312.

14. Monte Carlo Method. Wikipedia. http//en.wikipedia.org/wiki/Monte_Carlo_method. 